\title{
On the Construction of Molaei's Generalized Hypergroups
}

\author{
Nosratollah Shajareh Poursalavati \\ Department of Pure Mathematics, Faculty of Mathematics and Computer, Mahani Mathematical Research Center, Shahid Bahonar University \\ of Kerman, Kerman, Iran
}

\section{Email address:}

salavati@uk.ac.ir

\section{To cite this article:}

Nosratollah Shajareh Poursalavati. On the Construction of Molaei’s Generalized Hypergroups. Science Journal of Applied Mathematics and Statistics. Vol. 5, No. 3, 2017, pp. 106-109. doi: 10.11648/j.sjams.20170503.12

Received: February 17, 2017; Accepted: April 20, 2017; Published: June 1, 2017

\begin{abstract}
The purpose of this paper is making a construction and generalization of Molaei's generalized groups by using construction of the Rees matrix semigroup over a polygroup $H$ and a matrix with entries in $H$. We call it "Molaei's generalized hypergroups" and we give some examples.
\end{abstract}

Keywords: Hypergroup, Polygroup, Molaei’s Generalized Hypergroup

\section{Introduction}

In [10] generalized groups or completely simple semigroups is introduced as a class of algebras of interest in physics and they are an interesting generalization of groups. In [1], it is proved the generalized groups are the completely simple semigroups. Aaújo and Konieczny used the Rees matrix semigroup, (see [8]) over a group and they showed that the Molaei's generalized groups are the completely simple semigroups. In this paper we change the group to the polygroup and we obtain a new construction, by using this construction we can define "Molaei's generalized hypergroup" and we give some examples.

Let $H$ be a non-empty set. A hyperoperation on $H$ is a function from $H \times H$ to $P^{*}(H)$, which $P^{*}(H)$ is the set of all non-empty subsets of $H$. A hypergroupoid is the couple $(H$, $*$, where $H$ is a non-empty set and "**" is a hyperoperation on $H$, i.e., *: $H \times H \rightarrow P^{*}(H)$. As usual, we write $a^{*} b=*(a, b)$, for all $a$ and $b$ in $H$. If $M$ and $N$ belong to $P^{*}(H)$ and $a$ be an element of $H$, we define:

$$
\begin{aligned}
& M * N:=\bigcup_{m \in M, n \in N} m * n, \quad M * a:=M *\{a\}, \\
& a * N:=\{a\} * N .
\end{aligned}
$$

The relational notation $M \approx N$ is used to assert that $M$ and $N$ have an element in common, i.e., $M \cap N$ is non empty set.

We recalled the following definitions: [3, 4, 6, 9]

1) the hyperoperatoin $* *$, is associative, if for every elements $a, b$ and $c$ of $H,\left(a^{*} b\right)^{*} c=a^{*}\left(b^{*} c\right)$;

2) the hypergroupoid $(H, *)$ is semihypergroup, if the hyperoperation ' $*$ ' is associative;

3) the hypergroupoid $(H, *)$ is quasihypergroup, if for all a of $H, a^{*} H=H^{*} a=H$;

4) the hypergroupoid $(H, *)$ is hypergroup if it is both quasihypergroup and semihypergroup,

$5)$ the hypergroup $(H, *)$ is polygroup if there exist a unique element $e$ in $H$, which for every $a$ in $H$,

$e^{*} a=a * e=\{a\}$, and there exists a unitary operation

${ }^{-1}: H \rightarrow H$, by $a$ maps to $a^{-1}$, which for every elements $a, b$ and $c$ in $H$, if $a$ be an element of $b * c$ then $b$ be an element of $a * c^{-1}$ and $c$ be an element of $b^{-1} * a$.

As usual, this polygroup is demonstrated by $\left\langle H, *, e,^{-1}\right\rangle$. We refer to $[2,5,6,7]$, for more details about polygroups.

Let $<H,{ }^{*}, e{ }^{-1}>$ be a polygroup and $K$ be a non-empty subset of $H$, we denoted $\mathrm{K}^{-1}=\left\{\mathrm{k}^{-1}: k\right.$ be an element of $\left.K\right\}$, it is easy to show that, the following axioms hold for every $a$ and $b$ in $H$,

$$
\left(a^{-1}\right)^{-1}=a, e^{-1}=e \text {, e } \epsilon\left(a * a^{-1}\right) \cap\left(a^{-1} * a\right),(a * b)^{-1}=b^{-1} * a^{-1} .
$$

\section{Molaei's Generalized Hypergroups}

In this section, we consider a polygroup and by using the Rees matrix semigroup's structure over polygroup, we construct a new structure and obtain three properties of this new structure. Theorem 2.1, 2.2 and 2.3 guide us inspire the definition of Molaei's generalized hypergroups.

Let $<H, *, e,^{-1}>$ be a polygroup and let $I, \Lambda$ be nonempty sets and $M$ be a map from $\Lambda \times I$ to $H$, by $\mathrm{M}(\lambda, \mathrm{i})=$ 
$\mathrm{m}_{\lambda \mathrm{i}}$

Assume that $\mathcal{M} \mathcal{G} \mathscr{H}(H ; I, \Lambda, M):=I \times H \times \Lambda$, We define the following hyper-composition:

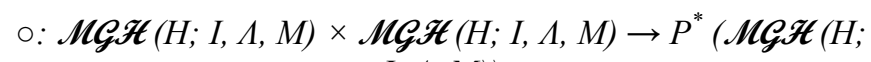
$I, \Lambda, M))$

$$
((i, x, \lambda),(j, y, \mu)) \mapsto(i, x, \lambda) \circ(j, y, \mu),
$$

which for all $i$ and $j$ in $I$, for all $x$ and $y$ in $H$ and for all $\lambda$ and $\mu$ in $\Lambda$,

$$
(i, x, \lambda) \circ(j, y, \mu):=\{i\} \times\left(x * m_{\lambda j} * y\right) \times\{\mu\} .
$$

Theorem 2.1. $\mathcal{M G} \mathscr{H}(H ; I, \Lambda, M)$ is a semihypergroup.

Proof. Let $i$ and $j$ in $I, \lambda$ and $\mu$ in $\Lambda$ and $a, b, c$ in $H$. Since $\left(a * m_{\lambda i} * b\right)$ is a non-empty subset of $H$, so

$$
\{\} \neq(i, x, \lambda) \circ(j, y, \mu) \in P^{*}(\mathcal{M G H}(H ; I, \Lambda, M)) .
$$

Therefore "O" is a hyperoperation. Now we check the associative property of hyperoperation " $\circ$ ”.

We have the following equations:

$$
\begin{aligned}
(i, a, \lambda) \circ((j, b, \mu) \circ(k, c, v) & \left.=(i, a, \lambda) \circ\left(\{j\} \times\left(b * m_{\mu k} * c\right) \times\{v\}\right)\right) \\
& =(i, a, \lambda) \circ \bigcup_{s \in b^{*} m_{\mu k}{ }^{*} c}(j, s, v) \\
& =\bigcup_{s \in b^{*} m_{\mu k}{ }^{*} c}(i, a, \lambda) \circ(j, s, v) \\
& =\bigcup_{s \in b^{*} m_{\mu k}{ }^{*} c}\{i\} \times(a * m \lambda j * s) \times\{v\} \\
& =\{i\} \times\left(\bigcup_{s \in b^{*} m_{\mu k} * c} a * m \lambda j * s\right) \times\{v\} \\
& =\{i\} \times((a * m \lambda j) *(b * m \mu k * c)) \times\{v\} \\
& =\{i\} \times((a * m \lambda j * b) * m \mu k * c)) \times\{v\} \\
& =\{i\} \times\left(\bigcup_{t \in a^{*} m_{\lambda j} * b} t * m \mu k * c\right) \times\{v\} \\
& =\bigcup_{t \in a^{*} m_{\lambda j}{ }^{*} b}\{i\} \times(t * m \mu k * c) \times\{v\} \\
& =\bigcup_{t \in a^{*} m_{\lambda j}{ }^{*} b}(i, t, \mu) \circ(k, c, v) \\
& =\left(\bigcup_{t \in a^{*} m_{\lambda j} * b}(i, t, \mu)\right) \circ(k, c, v) \\
& =(\{i\} \times(a * m \lambda j * b) \times\{\mu\}) \circ(k, c, v) \\
& =((i, a, \lambda) \circ((j, b, \mu)) \circ(k, c, v) \\
&
\end{aligned}
$$

Therefore, $\boldsymbol{M} \mathcal{G H}(H ; I, \Lambda, M)$ is a semihypergroup.

Theorem 2.2. For every element $(\mathrm{i}, \mathrm{a}, \lambda) \in \mathcal{M} \mathcal{G} \mathscr{H}(\mathrm{H} ; \mathrm{I}, \Lambda, \mathrm{M})$, there is a unique non-empty subset $\mathrm{E}(\mathrm{i}, \mathrm{a}, \boldsymbol{\lambda}) \subseteq \boldsymbol{M} \mathcal{G} \mathscr{H}(\mathrm{H} ; \mathrm{I}$, $\Lambda, M)$, such that for every element $(\mathrm{j}, \mathrm{b}, \mu)$ of $E(\mathrm{i}, \mathrm{a}, \lambda)$, implies

$(i, a, \lambda) \in[(i, a, \lambda) \circ(j, b, \mu)] \cap[(j, b, \mu) \circ(i, a, \lambda)]$. Moreover,

$$
E(i, a, \lambda)=\{i\} \times\left[\left(m_{\lambda i}{ }^{-1} * a^{-1} * a\right) \cap\left(a^{*} a^{-1} * m_{\lambda i}{ }^{-1}\right)\right] \times\{\lambda\} .
$$

Proof. Since $m_{\lambda \mathrm{i}}$ is an element of polygroup $H$, there exist $m_{\lambda \mathrm{i}}{ }^{-1}$, such that $e \in\left[\left(m_{\lambda i}{ }^{-1} * m_{\lambda i} \cap\left(m_{\lambda i} * m_{\lambda i}{ }^{-1}\right)\right]\right.$. Now, we have:

$$
\begin{aligned}
(i, a, \lambda) \circ\left(i, m_{\lambda i}^{-1}, \lambda\right) & =\{i\} \times\left(a * m_{\lambda i} * m_{\lambda i}^{-1}\right) \times\{\lambda\} \\
& \supseteq\{i\} \times(a * e) \times\{\lambda\}=\{i\} \times\{a\} \times\{\lambda\}=\{(i, a, \lambda)\} . \\
\text { Also }\{(i, a, \lambda)\} & =\{i\} \times\{a\} \times\{\lambda\}=\{i\} \times(e * a) \times\{\lambda\} \\
& \subseteq\{i\} \times\left(m_{\lambda i}^{-1} * m_{\lambda i} * a\right) \times\{\lambda\} \\
& =\left(i, m_{\lambda i}^{-1}, \lambda\right) \circ(i, a, \lambda) .
\end{aligned}
$$

Therefore, $\left(i, m_{\lambda i}{ }^{-1}, \lambda\right)$ is an element of $\mathrm{E}(i, a, \lambda)$.

If $(j, b, \mu)$ be an arbitrary element of $E(i, a, \lambda)$, then we have:

$$
\begin{aligned}
(i, a, \lambda) & \in[(i, a, \lambda) \circ(j, b, \mu)] \cap[(j, b, \mu) \circ(i, a, \lambda)] \\
& =\left[\{i\} \times\left(a * m_{\lambda j}^{*} b\right) \times\{\mu\}\right] \cap\left[\{j\} \times\left(b * m_{\mu i} * a\right) \times\{\lambda\}\right] .
\end{aligned}
$$


Therefore, $\mathrm{j}=\mathrm{i}$ and $\mu=\lambda$ and $\mathrm{a} \in\left(\mathrm{a} * \mathrm{~m}_{\lambda \mathrm{i}} * \mathrm{~b}\right) \cap\left(\mathrm{b} * \mathrm{~m}_{\lambda \mathrm{i}} * \mathrm{a}\right)$. Since,

$$
\begin{aligned}
& a \in\left(a * m_{\lambda i} * b\right) \Leftrightarrow b \in\left(a * m_{\lambda i}\right)^{-1} * a=m_{\lambda i}^{-1} * a^{-1} * a, \\
& a \in\left(b^{*} m_{\lambda i} * a\right) \Leftrightarrow b \in a *\left(m_{\lambda i} * a\right)^{-1}=a * a^{-1} * m_{\lambda i}^{-1},
\end{aligned}
$$

therefore, $b \in\left[\left(m_{\lambda i}^{-1} * a^{-1} * a\right) \cap\left(a * a^{-1} * m_{\lambda i}^{-1}\right)\right]$.

Conversely if $\mathrm{b} \in\left[\left(\mathrm{m}_{\lambda \mathrm{i}}{ }^{-1} * \mathrm{a}^{-1} * \mathrm{a}\right) \cap\left(\mathrm{a}^{*} \mathrm{a}^{-1} * \mathrm{~m}_{\lambda \mathrm{i}}{ }^{-1}\right)\right]$, then $(\mathrm{i}, \mathrm{b}, \lambda)$ is an element of $\mathrm{E}(\mathrm{i}, \mathrm{a}, \lambda)$ and the proof is complete.

Theorem 2.3. For every element $(i, a, \lambda) \in \mathcal{M} \mathcal{G} \mathscr{H}(H ; I, \Lambda, M)$, there is a non-empty subset $\mathcal{J}(\mathrm{i}, \mathrm{a}, \lambda) \in \mathcal{M} \mathcal{G} \mathscr{H}(\mathrm{H} ; \mathrm{I}, \Lambda, \mathrm{M})$, such that for all $\beta$ in $\mathcal{J}(i, a, \lambda)$

$$
[(i, a, \lambda) \circ \beta] \cap[\beta \circ(i, a, \lambda)] \approx E(i, a, \lambda) .
$$

Proof. Assume that $c=m_{\lambda \mathrm{i}}$, by choosing $\mathcal{J}(i, a, \lambda)=\{i\} \times\left(c^{-1} * a^{-1} * c^{-1}\right) \times\{\lambda\}$. which, it is a non-empty subset of $\mathscr{M \mathcal { G }} \mathscr{H}$ $(H ; I, A, M)$, we show that it satisfies the condition of Theorem. Since $H$ is a polygroup, hence, $\{a\}=a * e$ and $e \in c * c^{-1}$ and $e \in a^{*} a^{-1}$ then we have:

$$
e \in a^{*} a^{-1}=(a * e) * a^{-1}=\left(a * e^{*} a^{-1}\right) \subseteq\left(a *\left(c * c^{-1}\right) * a^{-1}\right)=\left(a * c * c^{-1} * a^{-1}\right) .
$$

Then $c^{-1 \in}\left\{c^{-1}\right\}=e * c^{-1} \subseteq\left(a * c * c^{-1} * a^{-1}\right) * c^{-1}=(a * c) *\left(c^{-1} * a^{-1} * c^{-1}\right)$.

Similarly,

$$
e \in a^{-1} * a=\left(a^{-1} * e\right) * a=\left(a^{-1} * e^{*} a\right) \subseteq\left(a^{-1} *\left(c^{-1} * c^{\prime} * a\right)=\left(a^{-1} * c^{-1} * c * a\right) .\right.
$$

Then $c^{-1}\left\{c^{-1}\right\}=c^{-1} * e \subseteq c^{-1} *\left(a^{-1} * c^{-1} * c * a\right)=\left(c^{-1} * a^{-1} * c^{-1}\right) * c * a$.

Also, $c^{-1} \in\left(c^{-1} * a^{-1} * a\right) \cap\left(a * a^{-1} * c^{-1}\right)$, hence, $\mathrm{c}^{-1}$ is an element of the following set

$$
\left[(a * c) *\left(c^{-1} * a^{-1} * c^{-1)}\right] \cap\left[\left(c^{-1} * a^{-1} * c^{-1}\right) * c * a\right] \cap\left[\left(c^{-1} * a^{-1} * a\right)\right] \cap\left[\left(a * a^{-1} * c^{-1}\right)\right],\right.
$$

then,

$$
\left(i, m_{\lambda i}{ }^{-1}, \lambda\right) \in[(i, a, \lambda) \circ \mathcal{J}(i, a, \lambda)] \cap[\mathcal{J}(i, a, \lambda) \circ(i, a, \lambda)] \cap E(i, a, \lambda) .
$$

Let $\beta=(i, x, \lambda) \in \mathcal{T}(i, a, \lambda)$, then $x \in c^{-1} * a^{-1} * c^{-1}$, hence $c^{-1} \in a^{*} c * x \cap x * c * a$, therefore $c^{-1}$ is an element of the set $[a * c * x] \cap[x * c * a] \cap\left[\left(c^{-1} * a^{-1} * a\right)\right] \cap\left[\left(a * a^{-1} * c^{-1}\right)\right]$, then

$$
\left(i, m_{\lambda i}{ }^{-1}, \lambda\right) \in[(i, a, \lambda) \circ(i, x, \lambda)] \cap[(i, x, \lambda) \circ(i, a, \lambda)] \cap E(i, a, \lambda) .
$$

and the proof is complete.

Theorem 2.1, 2.2 and 2.3 guidance us to follows for definition of a generalization of Molaei's generalized group.

Definition 2.1. A semihypergroup ( $\mathscr{H}$, o) is called Molaei's generalized hypergroup, if it satisfies in the following conditions:

(MGH1) $\forall h \in \mathscr{H}, \exists ! \mathcal{E}(h) \subseteq \mathscr{H}$, such that for every element $\alpha \in \mathcal{E}(h), h \in[h \circ \alpha] \cap[\alpha \circ h]$,

(MGH1) $\forall h \in \mathscr{H}, \exists \mathcal{J}(h) \subseteq \mathscr{H}$, such that for every element $\beta \in \mathcal{J}(h),[h \circ \beta] \cap[\beta \circ h] \approx \mathcal{E}(h)$,

(The symbole $\exists$ ! means there is a unique.)

Example 2.1. If $<H, *, e,{ }^{-1}>$ be a polygroup and let $I, A$ be non-empty sets and $M$ be a map from $\Lambda \times I$ to $H$ by $M(\lambda$, i) $=m_{\lambda \mathrm{i}}$. Then, by use Theorems 2.1, 2.2 and 2.3, $\boldsymbol{M} \mathcal{G} \mathscr{H}(H$; $I, \Lambda, M):=I \times H \times \Lambda$, with hyperoperation " $O$ " is a Molaei's generalized hypergroup.

Example 2.2. Every polygroup is a Molaei's generalized hypergroup. If $<H, *, e,^{-1}>$ be a polygroup, it is semihypergroup and for every element $h \in H$,

$$
\mathcal{E}(h)=\left(h^{*} h^{-1}\right) \cap\left(h^{-1} * h\right) \text { and } \mathcal{J}(h)=\left\{h^{-1}\right\} .
$$

Example 2.3. Every Molaei's generalized group is a Molaei's generalized hypergroup. If $G$ be a Molaei's generalized group, we consider the hyperoperation $x$ $*_{y}=\{x y\}$, then $(G, *)$ is a semihypergroup and for every $h \in$ G,

$$
\mathcal{E}(h)=\{e(h)\} \text { is unique and } \mathcal{J}(h)=\left\{h^{-1}\right\} .
$$

\section{Conclusion}

This paper deal with one of the newest construction of a generalization of hypergroups. We changed the group to the polygroup in the structure of Rees matrix semigroup and we obtained a new construction. By using this construction we defined "Molaei's generalized hypergroup" and we gave some examples.

\section{References}

[1] J. Aaújo and J. Konieczny, Molaei's generalized groups are completely simple semigroups, Bul. Inst. Politeh. Jassy, Sect. I. Mat. Mec. Teor. Fiz. 48(52) (2002) 1-5.

[2] H. Aghabozorgi, B. Davvaz and M. Jafarpour, Solvable polygroups and derived subpolygroups, Communications in Algebra, 41(8) (2013) 3098-3107. 
[3] S. D. Comer, Polygroups derived from cogroups, J. Algebra, 89 (1984) 397-405.

[4] P. Corsini, Prolegomena of Hypergroup Theory, Aviani Editore, 1993.

[5] B. Davvaz, Isomorphism theorems of polygroups, Bull. Malays. Math. Sci. Soc. (2), 33(3) (2010) 385-392.

[6] B. Davvaz, Polygroup Theory and Related Systems, World Scienti_c Publishing Co. Pte. Ltd., Hackensack, NJ, 2013.
[7] M. Jafarpour, H. Aghabozorgi and B. Davvaz, On nilpotent and solvable polygroups, Bulletin of Iranian Mathematical Society, 39(3) (2013) 487-499.

[8] J. M. Howie, Fundamentals of semigroup theory, Oxford University Press, New York, 1995.

[9] F. Marty, Sur une generalization de la notion de groupe, 8iem congres Math. Scandinaves, Stockholm, (1934) 45-49.

[10] M. R. Molaei, Generalized groups, Bulet. Inst. Politehn. Ia,si Sect,. I, 45(49) (1999) 21-24. 